\title{
Electrical properties of in-plane-implanted graphite nanoribbons
}

B. C. Camargo, R. F. de Jesus, B. V. Semenenko, and C. E. Precker

Citation: Journal of Applied Physics 122, 244302 (2017); doi: 10.1063/1.4995223

View online: https://doi.org/10.1063/1.4995223

View Table of Contents: http://aip.scitation.org/toc/jap/122/24

Published by the American Institute of Physics

\section{Articles you may be interested in}

Determination of electrical properties of degraded mixed ionic conductors: Impedance studies with applied dc voltage

Journal of Applied Physics 122, 244101 (2017); 10.1063/1.5006062

Broadband acoustic focusing by cavity structures with phase manipulations

Journal of Applied Physics 122, 244501 (2017); 10.1063/1.4998223

Enhanced and one-way absorptance of $\mathrm{LiNiO}_{2}$ thin films in one-dimensional photonic crystals Journal of Applied Physics 122, 243104 (2017); 10.1063/1.5007818

A force-matching Stillinger-Weber potential for $\mathrm{MoS}_{2}$ : Parameterization and Fisher information theory based sensitivity analysis

Journal of Applied Physics 122, 244301 (2017); 10.1063/1.5007842

Acoustic Dirac degeneracy and topological phase transitions realized by rotating scatterers Journal of Applied Physics 123, 091703 (2018); 10.1063/1.5004073

Microrobot with passive diamagnetic levitation for microparticle manipulations

Journal of Applied Physics 122, 243901 (2017); 10.1063/1.5005032

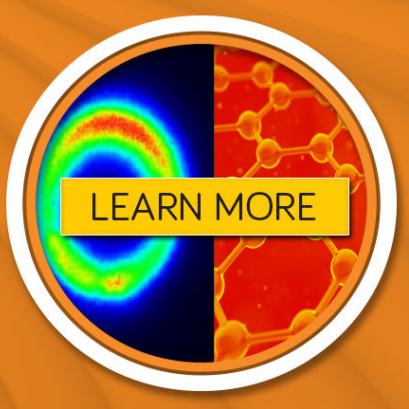




\title{
Electrical properties of in-plane-implanted graphite nanoribbons
}

\author{
B. C. Camargo, ${ }^{1,2, a)}$ R. F. de Jesus, ${ }^{3}$ B. V. Semenenko, ${ }^{2}$ and C. E. Precker $^{2}$ \\ ${ }_{1}^{1}$ Institute of Physics, Polish Academy of Sciences, Aleja Lotnikow 32/46, PL-02-668 Warsaw, Poland \\ ${ }^{2}$ Division of Superconductivity and Magnetism, Felix Bloch Institute for Solid State Physics, \\ Universitat Leipzig, Linnéstrasse 5, D-04103 Leipzig, Germany \\ ${ }^{3}$ Instituto Federal do Rio Grande do Sul, Campus Vacaria, 95200-000 Vacaria, RS, Brazil
}

(Received 10 July 2017; accepted 2 December 2017; published online 22 December 2017)

\begin{abstract}
We studied the effect of low energy $(30 \mathrm{keV})$ ionic implantation of $\mathrm{Ga}^{+}$in the direction parallel to the graphene planes (perpendicular to the c-axis) in oriented graphite ribbons with widths around $500 \mathrm{~nm}$. Our experiments have reproducibly shown a reduction of electrical resistance upon implantation consistent with the occurrence of ionic channeling in our devices. Our results allow for new approaches in the modulation of the charge carrier concentration in mesoscopic graphite. Published by AIP Publishing. https://doi.org/10.1063/1.4995223
\end{abstract}

\section{INTRODUCTION}

Graphite is a material composed by loosely stacked graphene planes, bound together by van der Waals forces. Due to its similarities with graphene, graphite attracts broad technological interest. Most notably, few-layer graphite has been the focus of increasingly larger research efforts in the past few years, partially due to its low electrical resistivity, high charge carrier mobility, as well as the possibilities of gap modulation and ballistic transport. ${ }^{1-4}$

One of the greatest challenges posed by mesoscopic graphite structures is the difficulty to control its charge carrier density. Due to its high native charge carrier concentration [ranging from $10^{18}$ to $10^{21} \mathrm{~cm}^{-3}$ (Ref. 4)], electrostatic doping is usually ineffective in samples with the thickness above few nanometers because of charge screening. In addition, conventional ionic implantation is often problematic due to the disorder introduced in the graphite structure by the highly energetic ions. ${ }^{5-7}$

In this letter, we address these issues by attempting ionic implantation parallel to the planes (perpendicular to c-axis) in narrow HOPG (highly oriented pyrolytic graphite) ribbons. Our results show a consistent resistivity reduction with the amount of implanted ions, suggesting implantation in this direction as a viable way to modulate the charge carrier density in the material.

\section{RESULTS AND DISCUSSION}

The samples studied here were HOPG ribbons extracted from two different bulk crystals: advanced ceramics ZYA HOPG (FWHM $0.5^{\circ}$ ) and Great Wall, Inc., GW (FWHM $\left.0.39^{\circ}\right) .{ }^{8,9}$ The bulk crystals had typical dimensions of $2 \mathrm{~mm}$ $\times 3 \mathrm{~mm}$ (in-plane) $\times 0.5 \mathrm{~mm}$ (c-axis) and room-temperature (RT) resistivities of $20 \mu \Omega \mathrm{cm}$ (ZYA) and $5 \mu \Omega \mathrm{cm}(\mathrm{GW})$. They presented a metallic-like behaviour $(\mathrm{dR} / \mathrm{dT}>0)$ with saturation at high $\mathrm{T}$, typical of well-graphitized bulk HOPG. ${ }^{10}$ This is shown in the inset of Fig. 1.

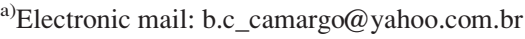

The ribbons were prepared as detailed in Refs. 11 and 12. In short, they were etched from a freshly cleaved HOPG surface with the $30 \mathrm{kV} \mathrm{Ga}^{+}$ion beam from a FEI dual-beam electron microscope. ${ }^{13}$ During the milling procedure, progressively smaller ion beam currents were used for etchings done near the ribbons edges. This was performed in order to polish the samples surfaces and limit the Ga diffusion in the material. SRIM (stopping range of ions in matter) simulations predict that, under these conditions, the lateral penetration of $\mathrm{Ga}^{+}$in graphite should remain under $20 \mathrm{~nm},{ }^{12,14}$ resulting in ribbons surrounded by, at most, a $20 \mathrm{~nm}$ layer of amorphous carbon. To negate milling damage to the top of the ribbon, the sample was covered in-situ with an $800 \mathrm{~nm}$-thick layer of insulating PdC, obtained by electron-beam induced deposition (EBID). ${ }^{12}$ The resulting samples had typical dimensions $20 \mu \mathrm{m} \times 500 \mathrm{~nm}$ (in-plane) $\times 5 \mu \mathrm{m}$ (along the c-axis-see the cartoon in the inset of Fig. 1). With a micro-manipulator, the ribbons were transferred to a $\mathrm{Si}$ substrate coated with a $300 \mathrm{~nm}$-thick layer of insulating $\mathrm{Si}_{2} \mathrm{~N}_{3}$. Due to their narrow in-plane width, the c-axis of the ribbons was oriented parallel to the substrate surface. Subsequently, the samples were soldered to the substrate with four EBID PdC staircase structures, in order to allow for an effective electrical contacting.

For the electrical addressing, the samples were submitted to a standard electronic lithography processes. In it, the devices were covered with a $1 \mu \mathrm{m}$-thick layer of PMMA resist, which was allowed to cure for $30 \mathrm{~min}$ at $180^{\circ} \mathrm{C}$ in a furnace. The electron-lithography writing was carried out with a dose of $120 \mu \mathrm{C} / \mathrm{cm}^{2}$ at an acceleration potential of $15 \mathrm{kV}$. After the developing process, the sample was exposed to a soft oxygen plasma $(15 \mathrm{~W})$ for $120 \mathrm{~s}$ to remove residues from the sample surface. This was followed by the sputtering deposition of a $10 \mathrm{~nm}$-thick adhesion layer of Pd, followed by a $90 \mathrm{~nm}$ layer of $\mathrm{Au}$. Pd was chosen as the adhesion layer due its low contact resistance and wide use in multigraphene samples (see, e.g., Ref. 15), as well as its good adherence to our $\mathrm{Si}_{2} \mathrm{~N}_{3}$ substrate. The liftoff of the resist was made with acetone. The resulting contacts short-circuited the sample along the c-axis, in an attempt to attain a homogeneous electrical current flow across the sample cross section (see the inset of Fig. 1). 


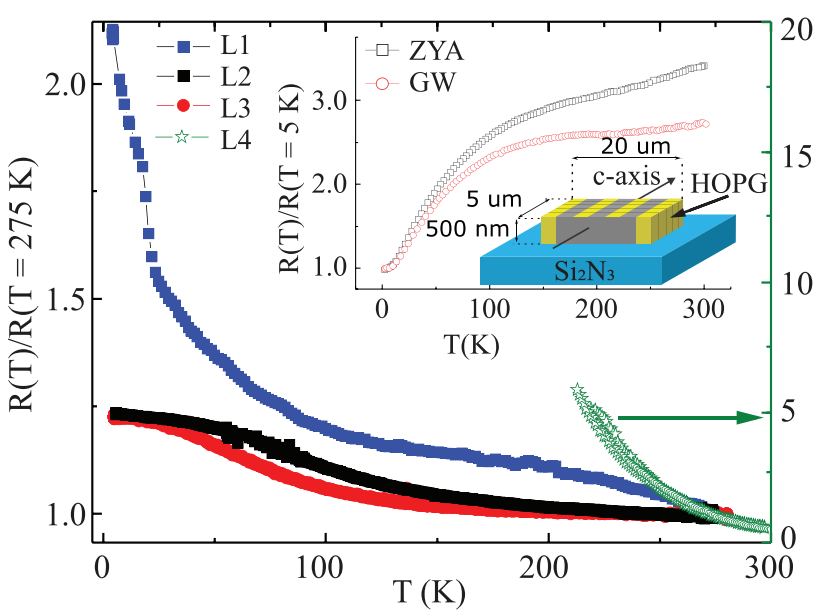

FIG. 1. Normalized R(T) measurements for four different ribbons. The left axis corresponds to samples L1, L2, and L3, while the right axis (green) corresponds to sample L4 (open symbols). The ice-temperature resistance $\mathrm{R}(\mathrm{T}=275 \mathrm{~K})$ of the samples was $2.3 \mathrm{k} \Omega$ for sample $\mathrm{L} 1,1.5 \mathrm{k} \Omega$ for $\mathrm{L} 2$, $0.3 \mathrm{k} \Omega$ for $\mathrm{L} 3$, and $15.0 \mathrm{k} \Omega$ for $\mathrm{L} 4$. The inset shows the curves for the bulk HOPG used in this work. The cartoon in the inset represents the geometry of samples L1-L4, as well as the typical dimensions of the devices. In it, lighter (yellow) patches correspond to the electrical contacts in the sample. The arrow points the sample c-axis direction.

In total, four samples were experimented. They are labeled L1, L2, L3, and L4. R(T) results are shown in Fig. 1. Samples L1, L2, and L3 were measured in the interval $4 \mathrm{~K}$ $\leq \mathrm{T} \leq 275 \mathrm{~K}$, while sample L4 was measured for $200 \mathrm{~K}$ $\leq \mathrm{T} \leq 300 \mathrm{~K}$ due to instrumentation limitations. All ribbons showed an insulating-like $\mathrm{R}(\mathrm{T})$ behavior $(\mathrm{dR} / \mathrm{dT}<0)$, in opposition to the metallic-like dependency observed in bulk HOPG (see the inset in Fig. 1). The ribbons roomtemperature (RT) resistivities were $6 \times 10^{4} \mu \Omega \mathrm{cm}$ for sample L1, $2.1 \times 10^{4} \mu \Omega \mathrm{cm}$ for sample L2, $0.7 \times 10^{4} \mu \Omega \mathrm{cm}$ for sample L3, and $20 \times 10^{4} \mu \Omega \mathrm{cm}$ for sample L4. These values are three to four orders of magnitude higher than those of bulk graphite ( $5 \mu \Omega \mathrm{cm}$ for $\mathrm{GW}$ and $20 \mu \Omega \mathrm{cm}$ for ZYA).

It would be natural to attribute the ribbons' insulatinglike behavior to an eventual disorder caused by $\mathrm{Ga}^{+}$ions during the milling process. However, SRIM simulations predict a diffusion of $\mathrm{Ga}$ in a region below $20 \mathrm{~nm}$ surrounding the ribbon, which is partially removed during the plasma etching in the lithography process. These suggest that disorder on the ribbons' surface cannot be held accountable for the high resistivity of the samples.

Instead, the insulating-like behavior and the enhanced resistivity of our devices can be understood in the context of finite-size effects. Mean-free paths of carriers in HOPG can reach values above tenths of micrometers, being in the same order of magnitude of the lateral width of our ribbons. ${ }^{4}$ Experiments in constricted HOPG demonstrate that the reduction of the lateral size of crystals below $1 \mu \mathrm{m}$ can result in an increase of the sample resistivity up to three orders of magnitude. ${ }^{11}$ Such an increase is non-linear with the constriction size and usually depends on the device studied. ${ }^{11}$ This aspect of our devices will be discussed elsewhere. ${ }^{16}$ In addition, the typical RT resistivities of the ribbons shown here agree with those observed in previous studies for similar samples. For example, in Refs. 11, 12, and 17, RT resistivities of HOPG ribbons vary between $10^{4} \mu \Omega \mathrm{cm}$ and $10^{6} \mu \Omega \mathrm{cm}$. Such devices have shown the same insulatinglike $\mathrm{R}(\mathrm{T})$ behavior found here when excited with electrical currents above few nano-Amperes.

After initial characterizations, our samples were covered with a $1 \mu \mathrm{m}$-thick layer of PMMA and a narrow window was patterned between the central sample electrodes with electronic lithography. The process was followed by developing and a soft plasma etching to remove PMMA residues and reduce the amorphous carbon layer coating our device. The samples were subsequently implanted with $30 \mathrm{kV} \mathrm{Ga}^{+}$ions, which were available in the same FEI dual beam electron microscope employed during the etching process. Due to the sample geometry, the implantation was performed with the ion beam parallel to the graphene planes (perpendicular to graphite's c-axis).

Results for device L2 are shown in Fig. 2. In it, each implanted dose corresponds to a fluence of $2.6 \times 10^{14}$ ions/ $\mathrm{cm}^{2}$. Measurements were done $e x$-situ with an AC resistance bridge operating at $\mathrm{f}=13 \mathrm{~Hz}$ and excitation currents $1 \mathrm{nA}$ $\leq \mathrm{I} \leq 10 \mathrm{nA}$ (each curve was measured at a constant excitation). All samples showed the same qualitative behavior and remained ohmic in the entire temperature range studied. Upon $\mathrm{Ga}^{+}$implantation, all devices initially became less resistive (up to one order of magnitude). Higher implanted concentrations (above $10^{15}$ ions $/ \mathrm{cm}^{2}$ ) caused a resistivity increase, which eventually led to the destruction of the devices. A diagram showing this effect for all our samples is presented in Fig. 3 and will be discussed further ahead.

Our results seem somewhat counter-intuitive, as $\mathrm{Ga}^{+}$ implantation in HOPG is reported to dramatically increase the sample resistance, rather than causing the pronounced decrease observed. For example, experiments performed on thin HOPG films with the ion beam parallel to the sample c-axis have shown that implantations with doses as small as $5 \times 10^{11}$ ions $/ \mathrm{cm}^{2}$ result in the increase of sample resistance above an order of magnitude. ${ }^{5}$ However, this behavior is not universal and strongly depends on the type of ions used. Implantation of $\mathrm{HOPG}$ with $\mathrm{H}^{+}$(parallel to the sample caxis), for example, shows a weak (1\%) sample resistance

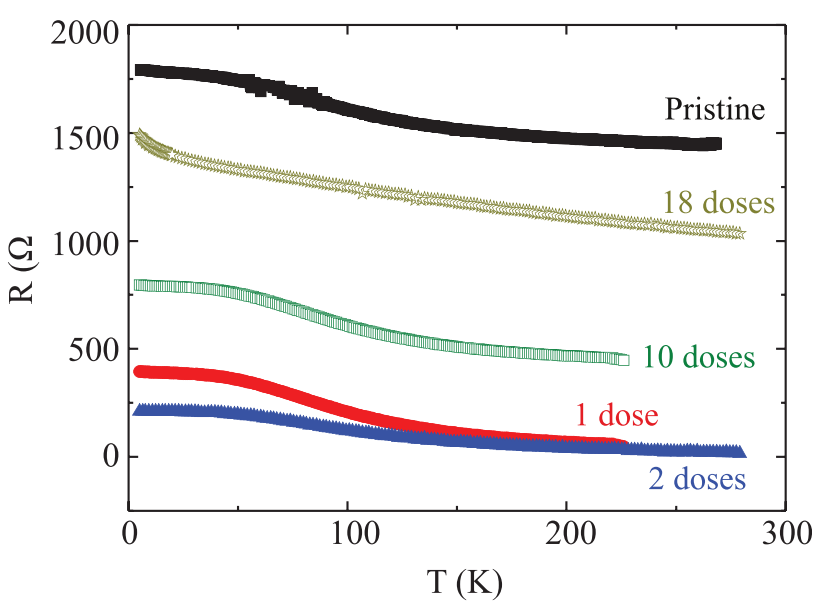

FIG. 2. R(T) measurements for sample L2 after consecutive ionic implantations. Note the resistance decrease (and subsequent increase) with the amount of implanted gallium. 

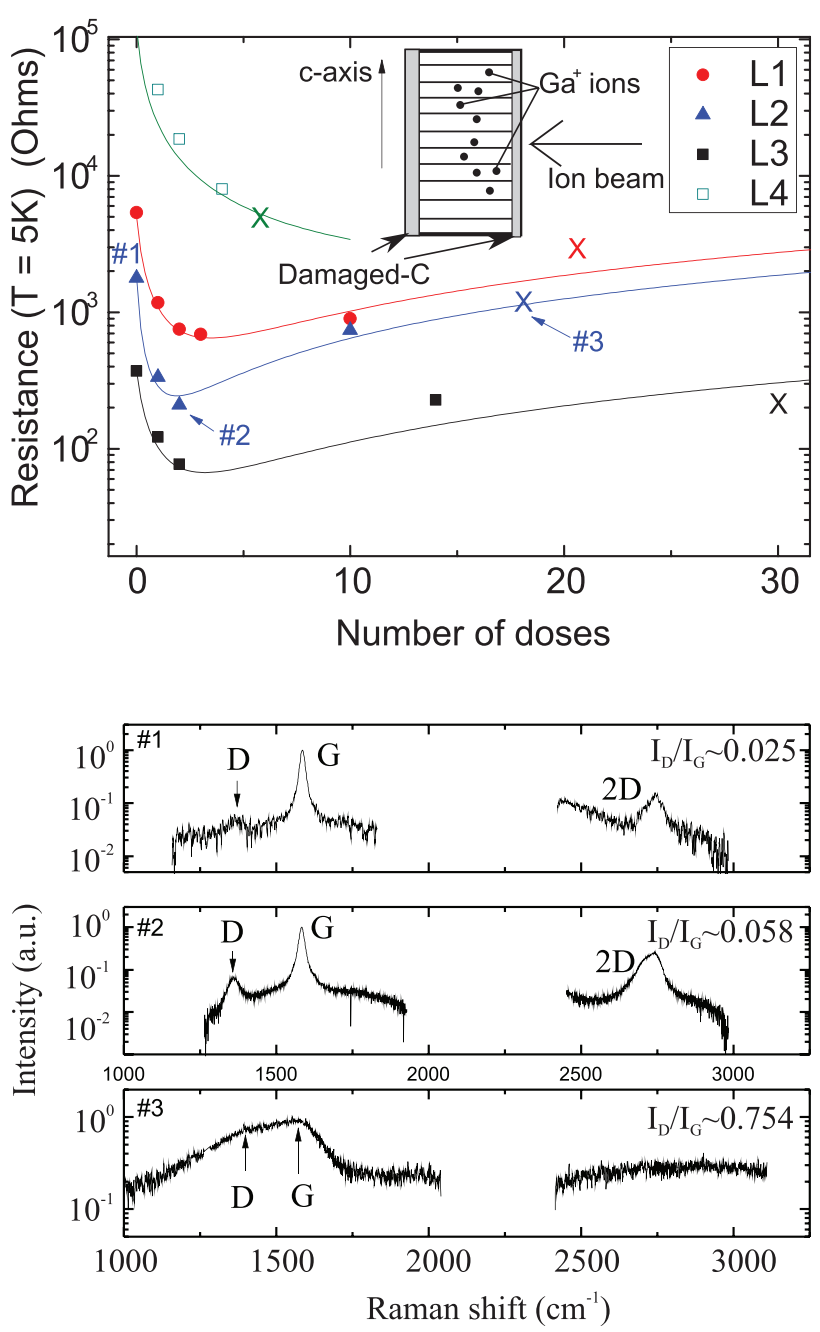

FIG. 3. Top panel: Low temperature resistance as a function of implanted $\mathrm{Ga}^{+}$ in our samples. Each dose corresponds to $2.6 \times 10^{14}$ ions $/ \mathrm{cm}^{2}$. The " $\mathrm{X}$ " corresponds to the last measured point before the samples were destroyed. The solid lines represent Eq. (1) with the parameters $\mathrm{R}_{0}=4.9 \times 10^{3} \Omega, \gamma=4.6 \times 10^{-3}$, and $\beta=90 \Omega \mathrm{cm}^{2}$ for sample $\mathrm{L} 1 ; \mathrm{R}_{0}=1.8 \times 10^{3} \Omega, \gamma=5.7 \times 10^{-3}$, and $\beta=62$ $\Omega \mathrm{cm}^{2}$ for sample $\mathrm{L} 2 ; \mathrm{R}_{0}=0.38 \times 10^{3} \Omega, \gamma=3.5 \times 10^{-3}$, and $\beta=20 \Omega \mathrm{cm}^{2}$ for sample L3; and $\mathrm{R}_{0}=120 \times 10^{3} \Omega, \gamma=5.4 \times 10^{-3}$, and $\beta=50 \Omega \mathrm{cm}^{2}$ for sample L4. The cartoon represents a ribbon cross-section showing how $\mathrm{Ga}^{+}$was implanted in the samples. In it, the large arrow shows the ion beam orientation relative to the graphene planes in the ribbon (black transverse lines). The gray areas represent the thin amorphous $\mathrm{C}$ layer covering the device, and the black dots the location of channeled $\mathrm{Ga}^{+}$. Bottom panel: Raman measurements of sample L2 at points \#1, \#2, and \#3. Measurements were performed with the laser incising perpendicular to the sample c-axis, with the electric field polarized along the graphene planes (similar to the $\mathrm{Ga}^{+}$implantation depicted in the top panel).

reduction for an implanted dose of $10^{13}$ ions $/ \mathrm{cm}^{2}$, followed by a monotonic resistance increase at higher fluences. ${ }^{6}$

The resistance increase observed in the literature is usually linked to the damage caused to in-plane chemical bondings in graphite. ${ }^{5,18}$ In our experiments, however, the ionic beam was oriented parallel to the sample planes (perpendicular to the c-axis). In this geometry, the implanted ions see atomic layers of $\mathrm{C}$ separated by distances about $3.35 \AA$, corresponding to the interplane distance in HOPG. These regions can allow ions to penetrate inside the material with minimal damage, in an effect known as ionic channeling.

It is well known that both axial and planar channeling can occur in higher quality HOPG. ${ }^{19-22}$ Experiments measuring the backscattering of $\mathrm{He}^{+}$ions in the last decade have shown that small misalignments between the sample and the ion beam do not considerably affect ionic channeling in the material. This applies to angles in the order of sample mosaicity and happens due to the presence of rotational faults (twist disorder) and mosaic spread in bulk graphite. ${ }^{18,19,21,23}$ In our experiments, the angle between the sample and the ion beam was controlled by a step motor with a precision of $0.1^{\circ}$ - which assured that misalignment angles remained below the sample mosaicity. Under these conditions, the implantation of heavy ions $\left(\mathrm{Ga}^{+}\right)$at the low energy regime $(30 \mathrm{keV})$ is likely to achieve a channeling condition. ${ }^{24}$

In order to verify the effect of ionic implantation on the sample structure, Raman measurements were performed. The experiments were carried out with the light incising perpendicular to the sample c-axis, with the electric field polarized along the graphene planes. Results for sample L2 at three implanted doses are shown in the lower panel of Fig. 3. Such doses correspond to the points marked in the resistanceimplantation diagram for all samples, which is shown in the upper panel of the same figure. The G-peak and 2D peaks on the Raman spectra are fingerprints of graphite, whereas the one labeled D is a signature of structural disorder. ${ }^{25}$ The curve \#1 shows the presence of the D-peak due to the disordered carbon layer surrounding the sample (presumably formed during the milling process). For subsequent doses (curves \#2 and \#3), the D-peak intensity gets higher as it overlaps with the G-peak. At this stage $\left(I_{G} / I_{D} \approx 1\right)$, the sample surface reaches the amorphization condition, no longer allowing measurements of the underlying graphite structure.

The Raman spectra can be compared with those previously obtained by Elman and Dresselhaus on HOPG implanted with different ions, albeit parallel to the c-axis. ${ }^{18,26}$ For example, Ref. 18 shows that the implantation of $100 \mathrm{keV}$ $\mathrm{He}^{+}$ions with a fluence of $1 \times 10^{14}$ ions $/ \mathrm{cm}^{2}$ is sufficient to produce the amorphization of the sample surface. At the same time, lesser energetic ions are shown to produce more damage to HOPG surface than their higher energetic counterparts. ${ }^{26}$

Considering these, it would be expected that the lower energy $\mathrm{Ga}^{+}$ions used here $(30 \mathrm{keV})$ would not produce an early surface amorphization at fluences of $10^{14} \mathrm{~cm}^{-2}$. This would happen due to the ions' larger energy transfer for collision, smaller scatter angle, smaller range, and larger defect density per volume when compared to lighter ions. ${ }^{27}$ These considerations suggest a channeling scenario for our graphite sample. As the implanted doses get higher, however, the channels are progressively destroyed as dechanneling and random scattering events become important. ${ }^{28,29}$

Hence, the ever increasing amount of damage can be conciliated with the sample resistance reduction by considering that our devices undergo a progressive amorphization, which competes with the doping caused by $\mathrm{Ga}$ atoms. The doping can be understood as a consequence of channeled $\mathrm{Ga}^{+}$ions acting as an interstitial linking between graphene planes in graphite, which donate electrons. ${ }^{23}$ Since the resistivity of graphite depends on the amount of charge carriers, ${ }^{10}$ this contribution reduces the sample resistance according to the inverse of the implanted dose. 
As the progressive damage increases with the amount of implanted ions, however, ionic channeling becomes suppressed. This results in a positive contribution to the sample resistance due to the scattering of upcoming ions, creating cascade process ${ }^{30}$ which increases the sample amorphization rate. Its contribution to the resistivity is non-trivial, but positive. Assuming that only implanted atoms act as scatterers, the dependency is linear. Considering these two competing effects, we propose the following phenomenological expression to describe the change of sample resistance with the amount of implanted ions:

$$
\mathrm{R}(\mathrm{d}) \approx \frac{\mathrm{R}_{0}}{1+\gamma \frac{\mathrm{d}}{n_{0}}}+\beta \times \mathrm{d} .
$$

In it, $d$ is the implanted dose, $R_{0}$ is the resistance of the pristine device, $n_{0}$ is the sample's native charge carrier concentration, $\beta$ is a constant representing the resistance gain due to the progressive amorphization, and $\gamma$ is a dimensionless constant related to the efficiency of the process. The first term in Eq. (1) describes the resistance reduction due to charge doping caused by ions, as the second term describes the disorderinduced (amorphization-related) resistance increase.

This tentative phenomenological model adjusts well to the experimental data with the use of two free parameters $\gamma$ and $\beta$ (except for sample L3, which did not have its $\mathrm{R}_{0}$ $(\mathrm{T}=5 \mathrm{~K})$ measured-see Fig. 1). The values of $\beta$ and $\gamma$ are within the same order of magnitude for all samples, suggesting that all devices undergo the same process. While the physical meaning of $\beta$ is not evident, the parameter $\gamma$ is directly linked to the efficiency of the implantation. It corresponds to the number of extra carriers added per implanted ion. Assuming ribbons with $n_{0} \approx 10^{11} \mathrm{~cm}^{-2}$ (as in bulk samples) gives an upper limit for $\gamma$ ranging between $3.5 \times 10^{-3}$ and $5.7 \times 10^{-3}$. This corresponds to an efficiency of about $0.5 \%$ for implanted doses in the range of $10^{14} \mathrm{~cm}^{-2}$. Such small values can be attributed to two factors: the relatively high fluence utilized and the lack of post-implantation thermal annealing. The reduction of the doping efficiency at high fluences is generally attributed to the creation of charge trap defects, which are always induced and hinder transport in the samples. ${ }^{30}$ This effect is usually remedied by a postimplantation thermal annealing, which was not done here. For example, it is shown in Refs. 30-32 that thermal annealing in different materials can improve the effective ionic doping above one order of magnitude by prompting recrystallization and solidary chemical bondings between the implanted element and the parent compound.

\section{CONCLUSIONS}

In conclusion, in this work, we have shown ionic doping parallel to planes in HOPG as a viable way to modulate the sample resistivity. Our results can be interpreted as the occurrence of ionic channeling in our samples in competition to amorphization induced by higher fluence ionic implantation. Our results suggest that ionic implantation perpendicular to the c-axis in HOPG modulates the sample charge carrier density while introducing much less damage when compared to implantation parallel to the sample c-axis. Our results point new routes towards the modulation of charge carriers in multigraphene devices and the functionalization of graphite nano objects.

\section{ACKNOWLEDGMENTS}

We would like to thank Professor Dr. Walter Escoffier for carefully reading the manuscript, Professor P. Esquinazi for insightful comments, and Professor Y. Kopelevich for constructive discussion and supplying the GW sample. This work was carried out with the financial support of $\mathrm{CNpQ}$ (Conselho Nacional de Desenvolvimento Cientifico e Tecnologico - Brazil) and by the Brazilian National Council for the Improvement of Higher Education (CAPES). B.C.C. acknowledges the financial support from the Polish National Science Center under Project No. UMO-2014/15/B/ST3/ 03889. We also acknowledge Erasmus Mundus Webb for the financial support.

${ }^{1}$ P. Esquinazi, J. Barzola-Quiquia, S. Dusari, and N. Garca, J. Appl. Phys. 111, 033709 (2012).

${ }^{2}$ C. C. Lui, Z. Li, K. F. Mak, E. Cappelluti, and T. F. heinz, Nat. Phys. 7, 944 (2011).

${ }^{3}$ E. V. Castro, K. S. Novoselov, S. V. Morozov, N. M. R. Peres, J. M. B. L. dos Santos, J. Nilsson, F. Guinea, A. K. Geim, and A. H. C. Neto, Phys. Rev. Lett. 99, 216802 (2007).

${ }^{4}$ N. Garcia, P. Esquinazi, J. Barzola-Quiquia, B. Ming, and D. Spoddig, Phys. Rev. B 78, 035413 (2008).

${ }^{5}$ J. Barzola-Quiquia, S. Dusari, G. Bridoux, F. Bern, A. Molle, and P. Esquinazi, Nanotechnology 21, 145306 (2010).

${ }^{6}$ A. Arndt, D. Spoddig, P. Esquinazi, J. Barzola-Quiquia, S. Dusari, and T. Butz, Phys. Rev. B 80, 195402 (2009).

${ }^{7}$ H. Miyazaki, S. Odaka, T. Sato, S. Tanaka, H. Goto, A. Kanda, K. Tsukagoshi, Y. Ootuka, and Y. Aoyagi, Appl. Phys. Express 1, 034007 (2008).

${ }^{8}$ See http://www.bjgreatwall.cn/ for Great Wall Co. Ltd., No. 10 Ronghua South Road, Yizhuang, Beijing, 100176, China.

${ }^{9}$ See http://www.momentive.com/ for information on Advanced Ceramics ZYA HOPG.

${ }^{10}$ B. T. Kelly, Physics of Graphite (Applied Science Publishers, 1981).

${ }^{11}$ J. Barzola-Quiquia, A. Ballestar, S. Dusari, and P. Esquinazi, "Experimental study of the intrinsic and extrinsic transport properties of graphite and multigraphene samples," in Graphene - Synthesis, Characterization, Properties and Applications, edited by J. Gong (InTech, 2011).

${ }^{12}$ A. Ballestar, J. Barzola-Quiquia, T. Scheike, and P. Esquinazi, New J. Phys. 15, 023024 (2013).

${ }^{13}$ See www.fei.com for FEI Inc., North America NanoPort, 5350 NE Dawson Creek Drive, Hillsboro, Oregon 97124, USA.

${ }^{14}$ See www.srim.org for Stopping and Range of Ions in Matter.

${ }^{15}$ B. K. Bharadwaj, D. Nath, R. Pratap, and S. Raghavan, Nanotechnology 27, 205705 (2016).

${ }^{16}$ C. E. Precker et al., "Influence of ballistic transport on the magnetoresistance of mesoscopic samples of natural graphite" (to be published).

${ }^{17}$ A. Ballestar, T. T. Heikkil, and P. Esquinazi, Supercond. Sci. Technol. 27, 115014 (2014).

${ }^{18}$ B. S. Elman, M. Shayegan, M. S. Dresselhaus, H. Mazurek, and G. Dresselhaus, Phys. Rev. B 25, 4142 (1982).

${ }^{19}$ M. S. Dresselhaus, R. Kalish, and E. Wieser, Ion Implantation in Diamond, Graphite and Related Materials (Springer, 1992).

${ }^{20}$ B. S. Elman, G. Braunstein, M. S. Dresselhaus, G. Dresselhaus, T. Venkatesan, and B. Wilkens, J. Appl. Phys. 56, 2114 (1984).

${ }^{21}$ D. Schroyen, M. Bruggeman, I. Dezsi, and G. Langouche, Nucl. Instrum. Methods Phys. Res., Sect. B 15, 341 (1986), ISSN: 0168-583X.

${ }^{22}$ R. Levi-Setti, G. Crow, Y. L. Wang, N. W. Parker, R. Mittleman, and D. M. Hwang, Phys. Rev. Lett. 54, 2615 (1985).

${ }^{23}$ X. Meng, S. Tongay, J. Kang, Z. Chen, F. Wu, S.-S. Li, J.-B. Xia, J. Li, and J. Wu, Carbon 57, 507 (2013), ISSN: 0008-6223.

${ }^{24} \mathrm{~B}$. Schmidt and K. Wetzig, Ion Beams in Materials Processing and Analysis (Springer-Verlag, Wien, 2013). 
${ }^{25}$ S. Reich and C. Thomsen, Philos. Trans. R. Soc. London, Ser. A 362, 2271 (2004), ISSN: 1364-503X.

${ }^{26}$ B. S. Elman, M. S. Dresselhaus, G. Dresselhaus, E. W. Maby, and H. Mazurek, Phys. Rev. B 24, 1027 (1981).

${ }^{27} \mathrm{M}$. Nastasi and J. W. Mayer, Ion Implantation and Synthesis of Materials (Springer, 2006).

${ }^{28}$ A. Gasparotto, A. Carnera, S. Acco, and A. L. Ferla, Nucl. Instrum. Methods Phys. Res., Sect. B 62, 356 (1992), ISSN: 0168-583X.
${ }^{29}$ T. Venkatesan, B. S. Elman, G. Braunstein, M. S. Dresselhaus, and G. Dresselhaus, J. Appl. Phys. 56, 3232 (1984).

${ }^{30}$ B. I. Kharisov, O. V. Kharissova, and U. O. Mendez, Radiation Synthesis of Materials and Compounds (CRC Press, 2016), Chap. 9.

${ }^{31}$ H. Hosono, H. Un'no, N. Ueda, H. Kawazoe, N. Matsunami, and H. Tanoue, Nucl. Instrum. Methods Phys. Res., Sect. B 106, 517 (1995), ISSN: 0168-583X. ${ }^{32}$ C. Wagner and F. Burkhardt, Phys. Status Solidi A 47, 131 (1978), ISSN: 1521-396X. 